\title{
High Presence Remote Presentation in the Shared Immersive Virtual World
}

\author{
Tetsuro Ogi ${ }^{1,2}$, Toshio Yamada ${ }^{1}$, Michitaka Hirose ${ }^{2}$, Masahiro Fujita ${ }^{2}$, Kazuto Kuzuu ${ }^{2}$ \\ ${ }^{1}$ MVL Research Center, TAO \\ ${ }^{2} I M L$, The University of Tokyo \\ tetsu@iml.u-tokyo.ac.jp
}

\begin{abstract}
In this study, a remote presentation system that can be used in the shared immersive virtual world was developed. This system consists of several elemental technologies, such as the video avatar, a shared database, shared visualization tools and an i-mode interface, and can be used for both meeting-style and demonstration-style presentations. This system was applied to a presentation of collaborative research in the field of fluid dynamics analysis, and the effectiveness was evaluated.
\end{abstract}

\section{Introduction}

The art of presentation is one of the important skill to be acquired by researchers and scientists who wish to inform other people about their work. In the field of virtual reality research, the ability to provide a high quality demonstration is particularly important. The purpose of this study is realizing a high presence remote presentation in the shared immersive virtual world. Specifically, the intention is that both meeting-style and demonstration-style presentations can be conducted.

\section{Elemental Technologies}

In order to construct an effective remote presentation system that can be used in the shared virtual world, the following functions are required.

a. natural communication between remote users

b. sharing of presentation materials

c. demonstration capability in the shared virtual world

d. interaction with the virtual world at each site

In this study, a remote presentation system that integrated several elemental technologies was developed to meet these requirements.

\subsection{Video Avatar}

In order to realize natural communication in the shared virtual world, it is necessary that the remote user's figure can be represented with a high quality of presence. In this study, stereo video avatar technology was developed [1]. In this method, the user's figure is captured by a stereo video camera, and a three-dimensional video image of the user is created from the depth values at each site. These images are mutually transmitted to the companion site, and they are superimposed onto the shared virtual world. Since this method transmits a live video image of the user, the gestures and facial expressions used by remote users can be directly represented in the virtual world.

\subsection{Shared Database}

Next, in the remote presentation system, slide-based data such as PowerPoint or OHP sheets should be able to be shared between remote sites. In this system, shared database system named CCBASE (Cyber Communication data BASE) was developed and used to construct a framework for sharing presentation materials in the shared virtual world. CCBASE allows a user to access data such as the PowerPoint through the network and manage them in the shared immersive virtual world (Figure 1). In addition, since the application program is also stored in the database, the demonstration program can be started in the environment of the PowerPoint presentation.

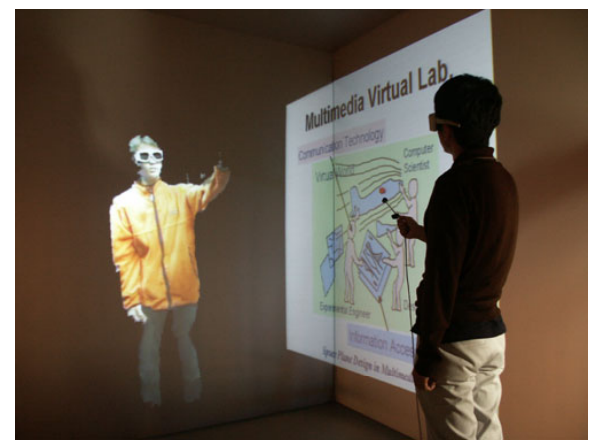

Figure 1. PowerPoint presentation in the shared virtual world 


\subsection{Shared Visualization Tool}

As an example of a demonstration program that can be used in the shared virtual world, a shared visualization tool named IVISOR (Interactive Visual Interface for threedimensional Stream with Outstanding Reality) was developed. IVISOR has several visualization functions for flow field data, such as streamlining, particle tracing, contour lines, surface layer rendering, and creating animations of these phenomena. In order to use IVISOR in the shared virtual world, the video avatar was also integrated (Figure 2).

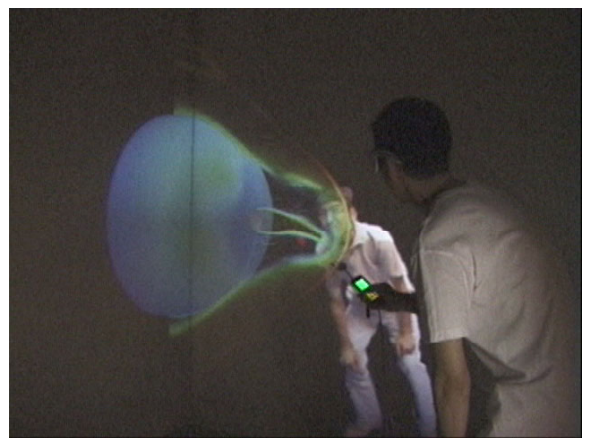

Figure 2. Shared demonstration using IVISOR

\section{4. i-mode Interface}

Finally, in order to realize an effective presentation and demonstration in the shared virtual world, it is necessary that the presenter and the audience can interact with the virtual world freely from both sites. In this system, the imode interface of a cellular phone was used for the interaction device. i-mode is an Internet access service provided by NTT DoCoMo, Inc., and it can be used to interact with a computer system through a web page. Since the i-mode interface communicates with the computer using a wireless network, both the users at the local site and at the remote site can access the communication server directly, and the inputted commands from both sites can be treated consistently.

\section{Remote Presentation Experiment}

In this study, a prototype of the remote presentation system was developed, by integrating the abovementioned elemental technologies. This system was used in the networked environment between CABIN at the University of Tokyo and COSMOS at the Gifu TechnoPlaza [2]. CABIN and COSMOS are CAVE-like multiscreen immersive projection displays that contain five and six screens respectively, and they are connected through the Japan Gigabit Network (Figure 3).

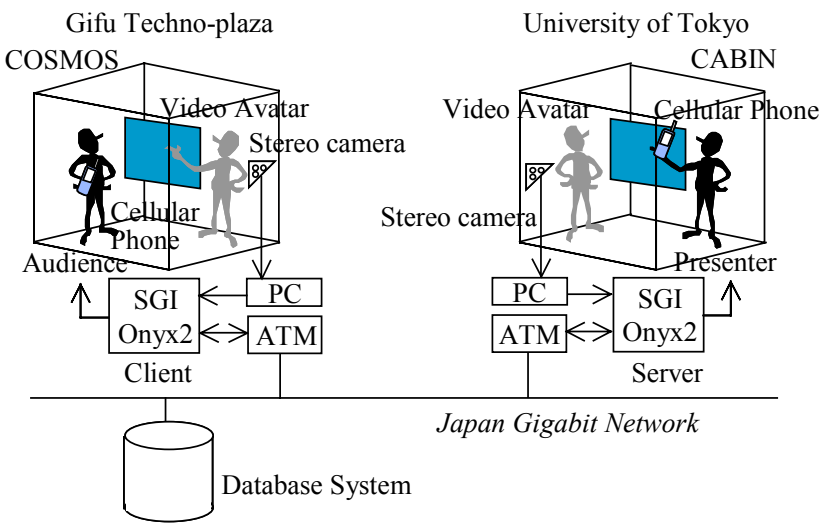

Figure 3 . System configuration of the
remote presentation environment

In this study, we asked researchers in the field of fluid dynamics analysis to use the prototype remote presentation system for their collaborative research between remote locations. Researchers at the University of Tokyo site gave a presentation to their collaborators at the Gifu Techno-Plaza site about their recent works using the shared immersive virtual environment. Their research topics included the numerical analysis of supersonic opposing jet flow and a visualization method for animation data using volume rendering. In this experiment, the users generally expressed a favorable impression of the system, and they told us after the experiment that they did not feel the need to meet with each other in the same location to carry out further discussions.

\section{Conclusions}

In this study, the framework for a remote presentation system using the broadband network was constructed. This system integrated several elemental technologies, such as the video avatar, a shared database, shared visualization tools and an i-mode interface. An experiment was conducted using this system in which we staged a remote presentation using the networked immersive projection displays that are available at the Tokyo and Gifu sites, and the effectiveness of this system was evaluated.

\section{References}

[1] T. Ogi, T. Yamada, M. Kano, M., Hirose, Immersive Telecommunication Using Stereo Video Avatar, Proceedings Virtual Reality 2001 Conference, pp.45-51, 2001

[2] M. Hirose, T. Ogi, S. Ishiwata, T. Yamada, Development and Evaluation of Immersive Multiscreen Display "CABIN", Systems and Computers in Japan, Vol.30, No.1, pp.13-22, 1999. 